\title{
Fearful Expressions Gain Preferential Access to Awareness During Continuous Flash Suppression
}

\author{
Eunice Yang, David H. Zald, and Randolph Blake \\ Vanderbilt University
}

\begin{abstract}
Rapid evaluation of ecologically relevant stimuli may lead to their preferential access to awareness. Continuous flash suppression allows assessment of affective processing under conditions in which stimuli have been rendered invisible due to the strongly suppressive nature of dynamic noise relative to static images. The authors investigated whether fearful expressions emerge from suppression into awareness more quickly than images of neutral or happy expressions. Fearful faces were consistently detected faster than neutral or happy faces. Responses to inverted faces were slower than those to upright faces but showed the same effect of emotional expression, suggesting that some key feature or features in the inverted faces remained salient. When using stimuli solely representing the eyes, a similar bias for detecting fear emerged, implicating the importance of information from the eyes in the preconscious processing of fear expressions.
\end{abstract}

Keywords: emotion, continuous flash suppression, fear, awareness

During our daily routines, we often encounter a plethora of objects and events, some of which can be highly relevant for our safety and even our survival. It is, therefore, highly adaptive to be able to rapidly detect and register signs of potentially dangerous objects or situations. Given this need, sensory processing systems may be automatically biased to detect affectively charged stimuli. For instance, in visual search tasks, negatively valenced stimuli are detected faster than neutral or positive stimuli among distractors (Fox et al., 2000). Behavioral findings further suggest that emotional stimuli can influence performance on certain tasks even when participants are not consciously aware of the stimuli. Studies using subliminal priming have shown that reaction time is faster when the prime and target are congruent in valence (Bargh, Chaiken, Govender, \& Pratto, 1992), and, similarly, subliminal priming effects have been reported with novel, nonrepresentational stimuli presented as targets (Murphy \& Zajonc, 1993; Wong \& Root, 2003). It is important to note that, under conditions of restricted attentional resources, emotional stimuli are able to reach conscious awareness even when neutral stimuli cannot (Anderson, 2005; Milders, Sahraie, Logan, \& Donnellon, 2006). The mechanism mediating this preferential access to awareness remains a matter of debate. It has often been discussed in terms of automatic preattentive processing. However, this view has been criticized, because effects are eliminated when attentional resources are directed elsewhere (see Palermo \& Rhodes, 2007, for review). Questions have also been raised regarding the quality of masking in some studies, suggesting the need for alternative methods of presenting stimuli outside of awareness.

Eunice Yang, David H. Zald, and Randolph Blake, Department of Psychology, Vanderbilt University.

This research was supported by National Institutes of Health Grant EY13358.

Correspondence concerning this article should be addressed to Eunice Yang, Psychology Department, Vanderbilt University, 111 21st Avenue South, Nashville, TN 37203. E-mail: eunice.yang@vanderbilt.edu
Binocular rivalry can temporarily prevent a visual stimulus from reaching awareness. Studies using binocular rivalry (and other forms of perceptual rivalry) have shown that the dynamics of this form of bistable perception are sensitive to the emotional overtones of the competing stimuli. Emotional facial expressions and aversely conditioned stimuli (gratings) predominate awareness relative to neutral stimuli in such studies (Alpers Ruhleder, Walz, Muhlberger, \& Pauli, 2005; Alpers \& Gerdes, 2007). Furthermore, unperceived facial expressions evoke amygdala activity during binocular rivalry (Pasley, Mayes, \& Schultz, 2004; Williams, Morris, McGlone, Abbott, \& Mattingley, 2004).

Recently Tsuchiya and Koch (2005) described a new procedure for erasing an ordinarily visible stimulus from visual awareness for extended durations; the technique is aptly called continuous flash suppression (CFS). Like binocular rivalry, this new technique involves dichoptic stimulation (i.e., dissimilar stimulation of the two eyes); in the case of CFS, a stimulus is prevented from reaching awareness by presenting strong dynamic noise to the opposing eye, allowing for long durations of suppression (Tsuchiya \& Koch, 2005). Because it provides stronger suppression than is typically seen with standard (static) binocular rivalry designs, CFS may be a more optimal technique for examining preconscious processing mechanisms. Jiang and He (2006) found that, with CFS, amygdala responsivity to suppressed fearful faces was comparable to its activity during perceived fearful faces and also that upright faces reached awareness faster than inverted faces (Jiang, Costello, \& He, 2007).

Intrigued by those observations, we have investigated whether fearful expressions rendered invisible by CFS emerge from suppression more quickly than do neutral or happy expressions, an outcome that would imply that emotionally charged stimuli have preferential access to awareness.

\section{Method}

\section{Participants}

Twelve observers ( 3 women) were recruited for Experiment 1, 14 (11 women) for Experiment 2, and 6 (3 women) for Experiment 
3. Participants had normal or corrected-to-normal acuity and good stereopsis, as assessed by standardized tests administered using a Bausch and Lomb Orthorator (Rochester, NY), and provided written informed consent. All were naïve to the purpose of the study.

\section{Procedure}

Stimuli consisted of four faces (two women) displaying fear, happy, and neutral expressions from the standard Ekman set of facial expressions (Ekman \& Friesen, 1976). Images were cropped to remove features outside of the face for Experiments 1 and 2. Everything but the eyes were cropped for Experiment 3 . To have strong initial suppression of the face/eye images, these images were normalized to $25 \%$ contrast (root mean square). Face/eye images were presented either upright or inverted. The CFS display consisted of grayscale Mondrian patterns normalized to $60 \%$ contrast (root mean square); the width and length of an individual rectangle in the Mondrian ranged from $.12^{\circ}$ to $.32^{\circ}$.

Stimuli were presented on the left and right halves of a video monitor ( $800 \times 600$ resolution; $120 \mathrm{~Hz}$ frame rate) and were viewed against a gray uniform background (see Figure 1 ). The stimuli were surrounded by fusion contours $\left(4.2^{\circ} \mathrm{x} 4.2^{\circ}\right)$ that served to promote stable binocular eye alignment, which was established for each individual by having that person carefully adjust the mirrors of the stereoscope while viewing dichoptic nonius stimuli that combine to produce a square frame only when the eyes are appropriately aligned. In the initial $1000 \mathrm{~ms}$, one eye was presented with a full contrast dynamic CFS display $\left(3.7^{\circ} \times 3.7^{\circ} ; 10 \mathrm{~Hz}\right)$ and the other eye viewed a face/eye image $\left(1.9^{\circ} \times 1.9^{\circ} / .5^{\circ} \times 1.8^{\circ}\right)$, the contrast of which was ramped up at a rate of $2 \%$ every $20 \mathrm{~ms}$, thereby avoiding abrupt transients. Once the face/eye image reached full contrast (approximately $1 \mathrm{~s})$, the contrast of the CFS target linearly decreased in contrast at a rate of $2 \% / 100 \mathrm{~ms}$ for the next $5,100 \mathrm{~ms}$. The eyes viewing the dynamic Mondrian and face/eye stimuli were randomized across trials.

In Experiment 1 (face detection), a face was presented in a random location within the square region corresponding to the rival target on the opposing half of the monitor, and observers pressed a key as soon as any part of the face stimulus emerged into dominance. On $30 \%$ of trials, no face was presented; on these trials, observers should withhold responses. These catch trials were terminated once the contrast of the CFS display reached 0 . Experiment 2 used an objective measure of perceptual awareness - a four alternative choice (4AFC) localization task. The face was presented within one of four quadrants and observers were instructed to press one of four buttons as quickly as possible once the quadrant containing a face, or any part of a face, was identified. Experiment 3 (eye detection) followed the procedure used in Experiment 1, except that the face stimuli were modified so that only the eyes were presented. In all experiments, trials terminated once responses were made, and reaction time (RT) and accuracy were recorded.

There were 60 trials for each condition (upright and inverted neutral, happy, and fearful expressions) with 15 repeats of each stimulus. Observers performed at least eight practice trials with neutral expressions not used in the experiments and took, on average, $30 \mathrm{~min}$ to complete an experiment.

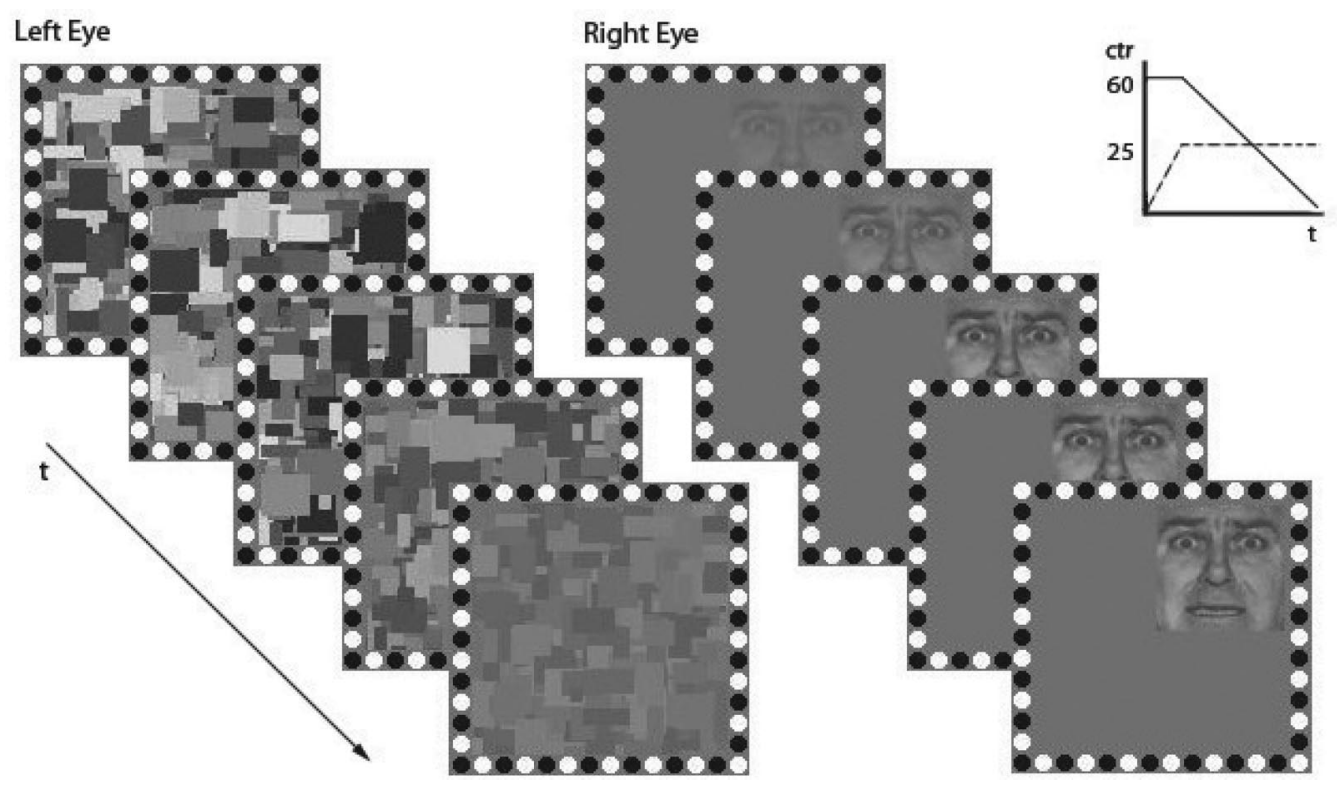

Figure 1. Example of a trial presentation. One eye initially viewed a high contrast continuous flash suppression (CFS) display (dynamic Mondrian); on most, but not all trials the other eye viewed a face image ramped up in contrast to a fixed value by the end of the first $1000 \mathrm{~ms}$. The contrast of the CFS target then gradually decreased until the observers indicated by button press the detection of the face (Experiment 1) or the location of a face (Experiment 2); if no face was seen, observers withheld their response. Figure in upper right corner depicts contrast of CFS (solid line) and face (dotted line) over the duration ( $t$ ) of a trial. 


\section{Results}

In Experiment 1, observers indicated the moment at which a face, or any part of the face, broke suppression to become visible. In the $30 \%$ of trials in which no face was presented, errors were rare; the false-alarm rate averaged $2.35 \%(S D=2.6 \%)$. All observers were careful, in other words, to respond on the basis of the emergence of a face into dominance.

Turning to the latency data, our results, like those of Jiang et al. (2007), show that RTs for upright faces were significantly faster than RTs for inverted faces; in our experiments, this was true for each category of emotional expression evaluated separately: neutral, $t(11)=6.41, p<.001$; happy, $t(11)=4.94, p<.001$; and fear, $t(11)=4.88, p<.001$. This is not surprising, for it is well established that faces are more salient when imaged in the upright orientation (face inversion effect). As for the effect of emotional expression, a within-subjects analysis of variance (ANOVA) revealed a main effect of expression, $F(1.6,17.64)=13.67, M S E=$ $.39, p<.001$, as illustrated by Figure 2a. Subsequent pairwise comparisons showed that RTs for detecting upright fearful faces $(M=2.98 \mathrm{~s}, S D=1.01)$ were significantly shorter than those for detecting upright faces displaying neutral $(M=3.25 \mathrm{~s}, S D=$ $1.01), t(11)=4.01, p=.002$, or happy expressions $(M=3.59$, $S D=.9), t(11)=3.1, p=.01$. We found the same pattern for inverted faces, with inverted fearful faces detected faster than inverted neutral $(M=3.69 \mathrm{~s}, S D=1.01), t(11)=3.41, p=.006$, and inverted happy faces $(M=4.06 \mathrm{~s}, S D=.94), t(11)=3.47$, $p=.005$, suggesting that some key feature or features in the inverted faces remained salient. We will return to this point shortly. Finally, there was a trend for RTs to happy faces to be slower than those for neutral faces, $t(11)=2.13, p=.06$.

We next tested whether differences in detecting emotional expressions could also be found using a forced-choice technique that provides a more objective measure of the threshold of perceptual awareness (Jakel \& Wichmann, 2006). In this second experiment, 2 out of 14 observers made errors greater than $10 \%$ of the time in correctly identifying the quadrant in which a face was presented, and their data were removed from the analysis. The mean error rate for the remaining 12 observers was $1.88 \%(S D=1.28 \%)$, which did not differ across facial expressions. As in Experiment 1, we

a

Experiment 1

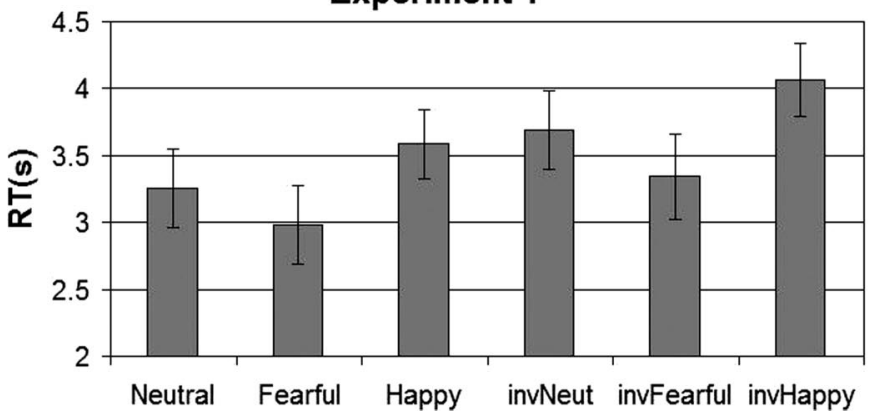

again see an inversion effect, with RTs to upright faces being faster than RTs to inverted faces: neutral, $t(11)=6.51, p<.001$; happy, $t(11)=5.92, p<.001 ;$ and fearful, $t(11)=3.9, p=.002$. Repeated measures ANOVA revealed a significant effect of facial expression, $F(2.1,23.09)=32.55, M S E=.11, p<.001$ (Figure 2b). RTs for detecting upright fearful faces $(M=2.06 \mathrm{~s}, S D=$ .71) were shorter than those for detecting upright neutral $(M=$ $2.33 \mathrm{~s}, S D=.77), t(11)=4.59, p=.001$, and upright happy faces $(M=2.64 \mathrm{~s}, S D=.81), t(11)=5.22 ; p<.001$. A similar pattern was again found with inverted faces: inverted fearful expressions ( $M=2.36 \mathrm{~s}, S D=.84)$ were detected faster than inverted neutral $(M=2.68 \mathrm{~s}, S D=.8), t(11)=3.62, p=.004$, and inverted happy expressions $(M=3.08 \mathrm{~s}, S D=.92), t(11)=6.04, p<.001$. Finally, observers detected happy expressions more slowly than neutral expressions, $t(11)=5.11, p<.001$.

The consistently briefer suppression durations for the fearful faces were not peculiar to one or two exemplars. Across the two experiments, all four fearful faces yielded shorter RTs than their neutral counterparts for seven out of eight possible instances (mean differences in Experiment $1=.93 \mathrm{~s}, .27 \mathrm{~s}, .19 \mathrm{~s},-.3 \mathrm{~s}$; Experiment $2=.39 \mathrm{~s}, .32 \mathrm{~s}, .16 \mathrm{~s}, .22 \mathrm{~s}$ ). The effect size (partial eta squared) for the interaction between expression and face identity was .22 in Experiment $1, F(4.56,50.12)=3.12, M S E=.62$, $p=.02$, observed power $=.81$, and .24 in Experiment $2, F(4.67$, $51.39)=3.53, M S E=.23, p=.01$, observed power $=.87$.

The results from Experiments 1 and 2 indicate that fearful faces gain preferential access to awareness even under conditions of strong suppression from dynamic images. All faces were equated for overall contrast. We also evaluated the visual similarity of the exemplars from the three categories of face images-neutral, happy, and fearful-in terms of their spatial frequency and orientation content. To accomplish this, we coded the features comprising each grayscale face image using 36 oriented Gaussianderivative filters ( 9 orientations $\times 4$ spatial scales) whose outputs were compared using the BOLAR model developed and validated by Zelinsky (2003). This model generates a similarity index for pairs of images that ranges from 0 (maximum dissimilarity in feature content) to 1.0 (identical in feature content). The similarity indices for the three pairwise comparisons were the following:

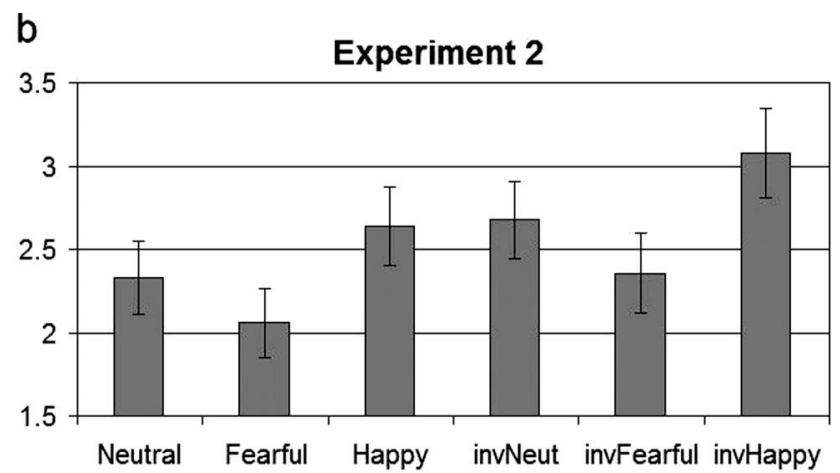

Figure 2. Results of Experiment 1 and 2. Mean reaction times (RT; seconds [s]) for conditions where the facial expressions presented were neutral, fearful, or happy in an upright or inverted (inv) orientation. Reaction times in Experiment 1 (left panel) represent the time it took to detect a face in the display, and reaction times in Experiment 2 (right panel) represent the time it took to locate the quadrant in which a face was presented. Error bars represent standard error of the mean. 
neutral/fearful $=.81(S D=0.04)$; neutral/happy $=.78(S D=$ $0.04)$; fearful/happy $=.79(S D=0.03)$. This analysis reveals that all of the images were highly similar in terms of the spatial frequency and orientation content. What, then, empowers the fearful faces to overcome suppression more readily?

Some observers subjectively reported seeing the eyes before any other facial feature, for both upright and inverted faces. Moreover, the BOLAR analysis consistently showed that the eyes in the fearful faces were the most conspicuous feature relative to their neutral counterparts (see Figure 3 ). Intrigued by these observations, we investigated whether the robust detection of fearful expressions is partly accounted for by the information portrayed in the eyes. Using the same task and display as Experiment 1, but with stimuli consisting of only eyes, Experiment 3 revealed emotion effects consistent with those of the previous two experiments, $F(1.72,8.59)=29.17, M S E=.46, p<.001$. Detecting eyes that convey fear $(M=2.74 \mathrm{~s}, S D=.78)$ was reliably faster than detecting eyes extracted from neutral expressions $(M=3.45 \mathrm{~s}$, $S D=1.02), t(5)=3.95, p=.01$, or happy expressions $(M=$ $4.65 \mathrm{~s}, S D=.79), t(5)=6.7, p=.001$. In addition, mean $\mathrm{RT}$ in the happy condition was significantly slower in comparison to that of the neutral condition, $t(5)=5.56, p=.003$.

\section{Discussion}

Using continuous flash suppression, we found that fearful expressions are detected more quickly than neutral or happy expressions. Furthermore, we found that these results are consistent across tasks of simple detection (Experiment 1) and detection with confirmed location identification (Experiment 2). Our results add
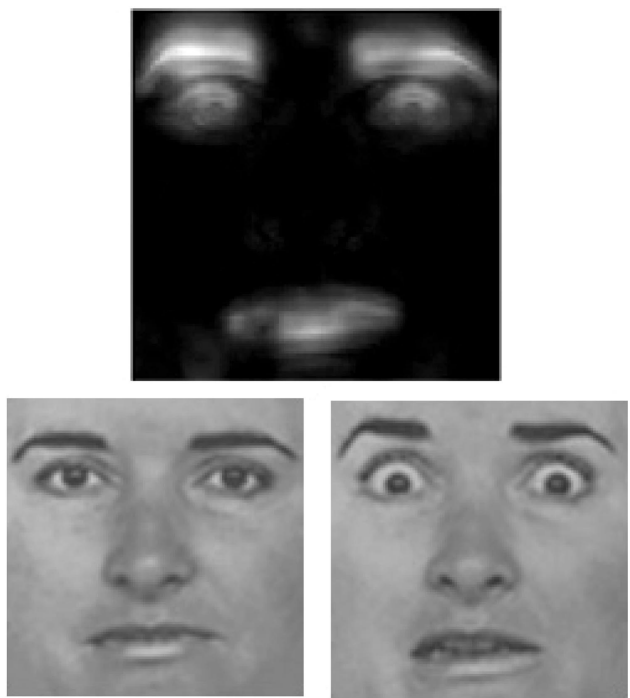

Figure 3. Difference map (top) between a pair of face images portraying neutral and angry expressions (bottom). The intensity values in the difference map are proportional to the dissimilarity between the two faces, computed from the BOLAR-derived representations (see text). The overall dissimilarity between these two faces is no greater than that for any other pairs of facial expressions, but this difference map highlights that the two differ primarily within the region of the eyes and, to a lesser extent, the mouth. Reprinted with permission from the Paul Ekman Group. to the growing evidence that negatively charged facial expressions gain preferential access to awareness.

The amygdala has been implicated in the rapid and reliable detection of behaviorally relevant stimuli based on human and animal studies (Phelps \& Ledoux, 2005; Zald, 2003). Amygdala lesion patients exhibit deficits in fear recognition (Adolphs et al., 2005). Other lesion studies have reported amygdala responsivity toward fearful or fear-conditioned faces when presented in subjects' blind field (Morris, DeGelder, Weisktrantz, \& Dolan, 2001) and during visual extinction (Vuilleumier et al., 2002). Neuroimaging studies have found similar results in amygdala activity using psychophysical techniques that manipulate subjects' awareness, including visual masking (Whalen, 1998; Whalen et al., 2004; Williams et al., 2006; but see Pessoa, Japee, Sturman, \& Ungerleider, 2006; Phillips et al., 2004) and binocular rivalry (Pasley et al., 2004; Williams et al., 2004).

Although a retino-collicular-pulvinar-amygdala pathway is hypothesized to be the neural substrate for the automatic processing of affective cues (Morris et al., 2001), the existence of this subcortical route has yet to be confirmed. Still, evidence from nonhuman primate single-unit studies have found amygdala response latencies to complex objects as fast as $60 \mathrm{~ms}$, almost twice as short as responses from the inferior temporal cortex, which has known feed-forward connections to the amygdala (Nakamura, Mikami, \& Kubota, 1992). Intraoperative electrophysiological studies in humans support the idea that responses to supraliminal emotional faces in the amygdala are faster than responses in cortical areas (Krolak-Salmon, Henaff, Vighetto, Bertand, \& Mauguiere, 2004), although at speeds that do not necessarily implicate a subcortical route. We know of only one intraoperative recording study that has utilized a flash suppression technique (Kreimen, Fried, \& Koch, 2002). In that study, 300-500 ms flash suppression eliminated amygdalar responses to famous faces. It remains to be seen whether single cell responses can be observed to emotional faces or under more prolonged CFS.

Our analysis of the spatial and orientation content of images revealed that the rapid detection of fear could not be attributed to global differences in spatial frequency or orientation. Rather, the main differences between images arose in a focal area of the image around the eyes. Consistent with the importance of this local feature, rapid detection of fear was also found with stimuli that consisted of eyes extracted from different expressions. This converges with Whalen and colleagues (2004) who found that the amygdala is responsive to masked images of eye whites of fearful expressions when compared with those of happy expressions. Accordingly, amygdala lesion patients are unable to naturally use information from the eyes in recognizing emotions (Adolphs et al., 2005).

Additionally, we found that neutral expressions (upright and inverted) were detected more quickly in comparison to happy expressions. Whalen (1998) proposed that amygdala activity might be inversely related to the quality of information regarding the nature of threat conveyed by stimuli. Because neutral expressions are more ambiguous in the nature of probable threat relative to happy expressions, they may take precedence over the less ambiguous safety signals conveyed by happiness.

In the present study, we validated the utility of a novel behavioral method for research in affective science. Specifically, CFS allows for the investigation of emotional processing that is independent of 
awareness and attention by reliably suppressing the perception of stimuli presented. To date the most common way of presenting subliminal stimuli has been to use extremely brief presentations, which often have to be presented for as little as $15 \mathrm{~ms}$ and followed by subsequent masking stimuli to avoid awareness. In contrast, the CFS procedure consistently rendered faces invisible for greater than 100 -fold longer periods of time. This extended suppression could potentially allow for longer periods of subliminal processing, which might in turn produce more robust behavioral effects in subliminal conditioning and priming experiments.

Our results imply that the salience of fearful expressions is not attributable to low-level features such as spatial frequency and orientation. Our image analyses, however, revealed that fearful faces are perceptually different (e.g., larger eye whites) in comparison to other expressions, and these cues may aid in detection during CFS. Further experiments are necessary to evaluate the salience of this facial attribute as well as to replicate our results with a wider range of emotionally laden stimuli.

\section{References}

Adolphs, R., Gosselin, F., Buchanan, T. W., Tranel, D., Schyns, P., \& Damasio, A. R. (2005). A mechanism for impaired fear recognition after amygdala damage. Nature, 433, 68-72.

Alpers, G. W., Ruhleder, M., Walz, N., Muhlberger, A., \& Pauli, P. (2005). Binocular rivalry between emotional and neutral stimuli: A validation using fear conditioning and EEG. International Journal of Psychophysiology, 57, 25-32.

Alpers, G. W., \& Gerdes, A. B. M. (2007). Here is looking at you: Emotional faces predominate in binocular rivalry. Emotion, 7, 495-506.

Anderson, A. K. (2005). Affective influences on the attentional dynamics supporting awareness. Journal of Experimental Psychology General, 134, 258-281.

Bargh, J. A., Chaiken, S., Govender, R., \& Pratto, F. (1992). The generality of the automatic attitude activation effect. Journal of Personality and Social Psychology, 62, 893-912.

Ekman, P., \& Friesen, W. V. (1976). Pictures of facial affect. Palo Alto, CA; Consulting Psychologists.

Fox, E., Lester, V., Russo, R., Bowles, R., Pichler, A., \& Dutton, K. (2000). Facial expressions of emotion: Are angry faces detected more efficiently? Cognition \& Emotion, 14, 61-92.

Jakel, F., \& Wichmann, F. A. (2006). Spatial four-alternative forced-choice method is the preferred psychophysical method for naive observers. Journal of Vision, 6, 1307-1322.

Jiang, Y., Costello, P., \& He, S. (2007). Processing of invisible stimuli: Advantage of upright faces and recognizable words in overcoming interocular suppression. Psychological Science, 18, 349-355.

Jiang, Y., \& He, S. (2006). Cortical responses to invisible faces: Dissociating subsystems for facial-information processing. Current Biology, 16, 2023-2029.

Kreiman, G., Fried, I., \& Koch, C. (2002). Single-neuron correlates of subjective vision in the human medial temporal lobe. Proceedings of the National Academy of Sciences, USA, 99, 8378-8383.

Krolak-Salmon, P., Henaff, M. A., Vighetto, A., Bertand, O., \& Mauguiere, F. (2004). Early amygdala reaction to fear spreading in occipital, temporal, and frontal cortex: A depth electrode ERP study in human. Neuron, 42, 665676.

Milders, M., Sahraie, A., Logan, S., \& Donnellon, N. (2006). Awareness of faces is modulated by their emotional meaning. Emotion, 6, 10-17.

Morris, J. S., DeGelder, B., Weisktrantz, L., \& Dolan, R. J. (2001). Differential extrageniculostriate and amygdala responses to presentation of emotional faces in a cortically blind field. Brain, 124, 1241-1252.

Murphy, S. T., \& Zajonc, R. B. (1993). Affect, cognition, and awareness: Affective priming with optimal and suboptimal stimulus exposures. Journal of Personality and Social Psychology, 64, 723-739.

Nakamura, K., Mikami, A., \& Kubota, K. (1992). Activity of single neurons in the monkey amygdala during performance of a visual discrimination task. Journal of Neurophysiology, 67, 1447-1463.

Palermo, R., \& Rhodes, G. (2007). Are you always on my mind? A review of how face perception and attention interact. Neuropsychologia, 45, 75-92.

Pasley, B. N., Mayes, L. C., \& Schultz, R. T. (2004). Subcortical discrimination of unperceived objects during binocular rivalry. Neuron, 42, 163-172.

Pessoa, L., Japee, S., Sturman, D., \& Ungerleider, L. G. (2006). Target visibility and visual awareness modulate amygdala responses to fearful faces. Cerebral Cortex, 16, 366-375.

Phelps, E. A., \& LeDoux, J. E. (2005). Contributions of the amygdala to emotion processing: From animal models to human behavior. Neuron, $48,175-187$.

Phillips, M. L., Williams, L. M., Heining, M., Herba, C. M., Russell, T., Andrew, C., et al. (2004). Differential neural responses to overt and covert presentations of facial expressions of fear and disgust. Neuroimage, 21, 1484-1496.

Tsuchiya, N., \& Koch, C. (2005). Continuous flash suppression reduces negative afterimages. Nature Neuroscience, 8, 1096-1101.

Vuilleumier, P., Armony, J. L., Clarke, K., Husain, M., Driver, J., \& Dolan, R. J. (2002). Neural response to emotional faces with and without awareness: Event-related fMRI in a parietal patient with visual extinction and spatial neglect. Neuropsychologia, 40, 2156-2166.

Whalen, P. J. (1998). Fear, vigilance, and ambiguity: Initial neuroimaging studies of the human amygdala. Current Directions in Psychological Science, 7, 177-188.

Whalen, P. J., Kagan, J., Cook, R. G., Davis, F. C., Kim, H., Polis, S., et al. (2004). Human amygdala responsivity to masked fearful eye whites. Science, 306, 2061.

Williams, L. M., Liddell, B. J., Kemp, A. H., Bryant, R. A., Meares, R. A., Peduto, A. S., et al. (2006). Amygdala-prefrontal dissociation of subliminal and supraliminal fear. Human Brain Mapping, 27, 652-661.

Williams, M. A., Morris, A. P., McGlone, F., Abbott, D. F., \& Mattingley, J. B. (2004). Amygdala responses to fearful and happy facial expressions under conditions of binocular suppression. Journal of Neuroscience, 24, $2898-2904$

Wong, P. S., \& Root, J. C. (2003). Dynamic variations in affective priming. Consciousness and Cognition, 12, 147-168.

Zald, D. H. (2003). The human amygdala and the emotional evaluation of sensory stimuli. Brain Research Reviews, 41, 88-123.

Zelinsky, G. J. (2003). Detecting changes between real-world objects using spatiochromatic filters. Psychonomic Bulletin \& Review, 10, 533-555.

Received March 8, 2007

Revision received July 10, 2007

Accepted July 16, 2007 